\title{
Aproximaciones a la comprensión de la conducta pacífica en jóvenes escolares: una perspectiva desde la psicología positiva
}

Fernando Robert Ferrel-Ortega*

Magíster en Psicología clínica. Profesor investigador, Facultad de Psicología,

Universidad Cooperativa de Colombia, Santa Marta, Colombia.

Correo electrónico:

fernando.ferrel@campusucc.edu.co

Recibido: 10 de octubre del 2014 Aprobado: 11 de noviembre del 2015

Cómo citar este artículo: Ferrel-Ortega, F. R. (2015). Aproximaciones a la comprensión de la conducta pacífica en jóvenes escolares: una perspectiva desde la psicología positiva. Pensando Psicología, 11(18), 153-161. doi: http://dx.doi.org/10.16925/pe.v11i18.1225

\section{Resumen}

Propósito: reflexionar y postular conceptos, categorías y estrategias para el desarrollo de la conducta pacífica, especialmente en niños y jóvenes, tanto en la educación como en la investigación, desde la psicología positiva, en cuanto a conductas saludables que promoverán una nueva cultura de paz. Tema: la importancia de la teoría del aprendizaje social de Albert Bandura y los aportes recientes de Richard Tremblay sobre el aprendizaje de la conducta violenta y pacífica en la primera infancia; también la reflexión sobre la educabilidad de la conducta pacífica prosocial desde una postura crítica, que cuestiona la aprobación social de la violencia por el statu quo. Desarrollo: la conducta violenta de uno o varios individuos, orientada a hacer daño a otras personas, es una preocupación constante en las ciencias sociales y humanas, así como en el individuo, la sociedad y el Estado. Hasta la fecha, las investigaciones acerca de los factores causales y el control y manejo de sus consecuencias se han orientado a sancionar, castigar e impedir la conducta violenta, sin preocuparse por descubrir y moldear aquella conducta que los seres humanos necesitan para vivir como tales, en sana convivencia, denominada aquí "conducta pacífica”, comportamiento opuesto a la conducta violenta. Conclusiones: el aprendizaje de la conducta pacífica tendría como consecuencia la sana convivencia social y el desarrollo humano saludable, dialogante y solidario, de manera que esto lleve al individuo a practicar relaciones interpersonales y sociales saludables y felices, como recursos que le dan sentido a la vida humana en todas sus actividades.

Palabras clave: aprendizaje social, conducta pacífica, psicología positiva. 


\title{
Approaches to Understanding Peaceful Conduct among Young Students: A Positive Psychology Perspective
}

\begin{abstract}
Purpose: To reflect and postulate concepts, categories and strategies for developing peaceful conduct, particularly among children and young people, both in education and research, from the perspective of positive psychology, in terms of healthy conduct that will promote a new culture of peace. Topic: The importance of the social learning theory of Albert Bandura and recent contributions by Richard Tremblay on the learning of violent and peaceful conduct in early childhood; also reflections on the ability to teach prosocial peaceful conduct from a critical posture, which questions social approval of violence by the status quo. Development: The violent conduct of one or more individuals, aimed at hurting other people, is a constant concern in the social and human sciences, as well as for individuals, society and the State. Until now, research into the causal factors and control and management of their consequences has aimed at sanctioning, punishing and impeding violent conduct, without trying to discover and mold that conduct that human beings need for their survival, in healthy coexistence, here denominated as 'peaceful conduct', or behavior that is the opposite of violent conduct. Conclusions: Learning peaceful conduct would result in healthy social coexistence along with healthy, human and supportive development characterized by dialogue, thus leading individuals to practice healthy and happy interpersonal and social relationships, as resources that give meaning to human life in all of its activities.
\end{abstract}

Keywords: social learning, peaceful conduct, positive psychology.

\section{Aproximações à compreensão do comportamento pacífico em jovens escolares: uma perspectiva a partir da psicologia positiva}

\section{Resumo}

Propósito: refletir e propor conceitos, categorias e estratégias para o desenvolvimento do comportamento pacífico, especialmente em crianças e jovens, tanto na educação quanto na pesquisa, a partir da psicologia positiva, sobre comportamentos saudáveis que promoverão uma nova cultura de paz. Tema: a importância da teoria da aprendizagem social de Albert Bandura e as contribuições recentes de Richard Tremblay a respeito da aprendizagem do comportamento violento e pacifista na primeira infância; também a reflexão sobre a educabilidade do comportamento pacífico prossocial a partir de uma postura crítica, que questiona a aprovação social da violência pelo statu quo. Desenvolvimento: o comportamento violento de um ou vários indivíduos, orientado a fazer mal a outras pessoas, é uma preocupação constante nas ciências sociais e humanas, bem como no indivíduo, na sociedade e no Estado. Até o momento, as pesquisas acerca dos fatores causais e do controle e gestão das suas consequências vêm sendo orientadas a sancionar, castigar e impedir o comportamento violento sem se preocupar em descobrir e moldar aquele comportamento que os seres humanos precisam para viver como tais, numa convivência sã, denominada aqui 'comportamento pacífico', comportamento oposto ao violento. Conclusões: aprendizagem do comportamento pacífico teria como consequência a convivência social sã e o desenvolvimento humano saudável, dialogante e solidário, de maneira que isso leve o indivíduo a praticar relações interpessoais e sociais saudáveis e felizes, como recursos que dão sentido à vida humana em todas as suas atividades.

Palavras-chave: aprendizagem social, comportamento pacífico, psicologia positiva. 


\section{Ojo por ojo... y la humanidad quedará ciega Mahatma Ghandi, 1972}

\section{Introducción}

La conducta violenta del ser humano es aquella que se realiza con la intención y motivación de hacer daño a otra persona, alejada de toda casualidad o circunstancia casual. Es decir, está orientada a ocasionar un perjuicio físico, psicológico o material a otra persona.

Muchas han sido las perspectivas teóricas que han pretendido identificar las causas y consecuencias de este tipo de comportamiento, lo que ha hecho imposible su explicación y por tanto, su control. En efecto, el origen de este tipo de comportamiento es multicausal, pues intervienen factores macrosociales (históricos, culturales, económicos, políticos e ideológicos) y microsociales (comunidad, familia e individuo). Adentrarse en ellos no es el fin de este artículo, ni mucho menos desde la perspectiva de la psicología como ciencia.

En este escrito solo se refiere la perspectiva psicológica para la explicación y la comprensión de la conducta violenta en los seres humanos, en la medida en que su presencia no solo altera la sana convivencia que debe existir entre las personas y su comunidad, sino también porque afecta el desarrollo del individuo como persona, como ser humano.

Los violentólogos de todos los tiempos han destacado los aspectos macrosociales como causantes de la violencia humana y no se equivocan. Sin embargo, las soluciones han sido precisamente fomentar una violencia mayor y bajo control del Estado. La consigna de acabar con la violencia ejerciendo mayor violencia parece ser la política y filosofía del Estado y la cultura en la que el indivuduo crece, estudia, vive en familia y comunidad, trabaja y percibe el futuro. No aparece otra alternativa. A continuación se ofrecen algunos ejemplos característicos de esta situación en la sociedad.

En reuniones políticas, sociales o festividades, regionales o nacionales, el Estado busca garantizar la seguridad y bienestar de los ciudadanos, incrementando el "pie de fuerza militar", casi que con la intención de tener un policía por ciudadano, a fin de "protegerlo". Hacerlo así es, desde esta perspectiva, un sinónimo de paz, seguridad y bienestar. No hay otra alternativa diferente.

De igual forma, ante cada manifestación social de ciudadanos que reclaman demandas reivindicativas o derechos específicos, el Estado responde con la violencia de las fuerzas armadas del Estado, encargado de reprimir y aplacar dicho comportamiento social que consideran que violenta el statu quo imperante. Como consecuencia, el movimiento se dispersa y se extingue, con lo cual consideran haber actuado con éxito frente a la conducta social violenta de la población reclamante. Sin embargo, no se fomenta ninguna otra alternativa comportamental efectiva para la consecución de logros de parte de estos actores sociales.

Así mismo, acontece frente a la actuación de la delincuencia, organizada o no, individual o de grupos, que amenaza la vida y los bienes/propiedades de los ciudadanos. A la actuación violenta de la delincuencia, el Estado la enfrenta con una mayor violencia contundente, con la represión militar, la condena judicial y el encierro carcelario de aquellos individuos o grupos de individuos que delinquen. Como consecuencia, se construyen más cárceles, pero los delincuentes se reproducen más de manera progresiva y en niveles alarmantes. De igual forma, no se vislumbra una solución alternativa a esta conducta violenta, solo el encierro y la oportunidad de salir y volver a delinquir.

De la misma manera, la máxima expresión de la violencia humana en sociedad es el estado de guerra interna a que llega una sociedad cuando un grupo humano se levanta en armas contra el Estado legalmente constituido. La búsqueda de solución frente a esta situación es también la puesta en marcha de una violencia mayor, planificada, organizada y cruel, a fin de someter a los alzados en armas. La respuesta no es diferente. El objetivo es aplastar la violencia de un sector con otra violencia de otro sector de la sociedad: violencia humana contra humanos. No se vislumbra tampoco otra alternativa de comportamiento social diferente al sometimiento incondicional y acrítico al statu quo imperante.

En el campo de la educación formal, en las instituciones educativas se observa algo parecido, en el aprendizaje y la convivencia social entre los miembros de la comunidad educativa (alumnos, profesores, autoridades educativas y padres de familia), pues también se presenta la conducta violenta. Violencia entre pares, violencia entre profesores y alumnos, violencia entre bandas juveniles que delinquen o se enfrentan con otras bandas similares, son algunas de sus manifestaciones. A diferencia del Estado, la respuesta de la escuela para afrontarla suele ser de dos tipos:

- Enfrentarla mediante la sanción, la represión, la baja calificación, la suspensión o expulsión del alumno o alumnos de la institución educativa. 
- Enfrentarla mediante una mayor formación en "valores humanos" tales como la responsabilidad, el respeto, la puntualidad, el cumplimento de las tareas signadas, la empatía y la solidaridad, entre otros.

Sin embargo, al parecer esto no es suficiente y el comportamiento violento en las escuelas continúa, y el bullying o violencia entre pares, es cada vez más generalizada, $y$ afecta la convivencia social entre los educandos provocando suicidios en algunos casos.

No es diferente lo que sucede en las familias de niños y jóvenes. Además de observar la violencia entre sus padres al afrontar algún problema entre ellos o por causas externas, también sufren diversos tipos de violencia de parte de sus padres o hermanos mayores con quienes conviven. Gritos, insultos, amenazas, castigos para estudiar, trabajo forzado y golpes, entre otros, son formas de conducta violenta con las que convive el adolescente y que muchas veces tienen que aceptar como hechos "normales" de la familia. Los padres, desde su posición de autoridad, proponen como alternativa solo el sometimiento y la pasividad del niño o joven frente al adulto con autoridad. No parece haber otra alternativa en la mayoría de los casos. Claro, padres que crecieron en un hogar violento tienden a ser violentos con sus propios hijos y, como consecuencia, padres violentos generarán hijos violentos.

De acuerdo con lo anterior, hay una tendencia mayoritaria de los niños y jóvenes a crecer en el marco de una cultura de violencia en todos los estratos y espacios de la sociedad, por lo que la conducta violenta de las personas se comporta como una epidemia y un verdadero problema de salud pública, el cual tiende a reproducirse rápidamente y amenaza la sana convivencia social, la salud, la educación, el desarrollo humano integral y la felicidad, recursos humanos del diario vivir (Organización Mundial de la Salud, 1986), los cuales deben ser alcanzados por todas las personas, en especial niños y jóvenes, con el respaldo del Estado.

Esta situación parece vislumbrar un futuro negativo frente a la presencia y expansión del comportamiento violento del ser humano en todas las esferas de su vida. Sin embargo, no debe ser así. Desde la perspectiva del autor, tanto el Estado como los estamentos educativos han equivocado las soluciones. Es evidente que frente a la conducta violenta no ha habido claridad respecto a su comprensión y mucho menos frente a su explicación y modificación; por tanto, se ha tratado de extinguir la conducta violenta sin presentar ni preocuparse por la conducta alterna - deseable socialmente- que deben aprender las nuevas generaciones. Para el autor esta conducta es precisamente la conducta opuesta que se denomina "conducta pacífica".

\section{¿Por qué ha sido y es vigente el uso de la conducta violenta en los seres humanos? ¿Cuál es la explicación?}

En primer lugar, cabe destacar que la violencia persiste, a nivel social e individual, porque sus consecuencias inmediatas son altamente efectivas. De acuerdo con Skinner (1953), psicólogo neoconductista creador del condicionamiento operante, si un comportamiento cualquiera es seguido de un estímulo aversivo (conducta "violenta" orientada a hacer daño), el comportamiento se extinguirá, y mientras más intenso sea el castigo, más rápido se extinguirá. En otras palabras, el efecto de un castigo o conducta violenta es altamente efectivo en la medida que elimina la conducta castigada. Hasta ahí todo estaría socialmente bien: eliminar la conducta indeseable es lo "deseable". Sin embargo, de acuerdo con el mismo Skinner, toda conducta que es seguida por consecuencias positivas inmediatas tiene grandes probabilidades de repetirse, lo cual indica que si el uso de la conducta violenta es seguida de consecuencias positivas inmediatas, muy probablemente se repetirá. Más aún, un comportamiento seguido de consecuencias positivas, es decir, constantemente reforzado, se repetirá, hará parte del repertorio de conducta específica del individuo, se convertirá en un hábito y se repetirá cada vez que este desee conseguir algo inmediato de otra persona. Así, la conducta violenta se reproducirá a la velocidad de la luz debido a su alta efectividad.

En segundo lugar, cabe precisar también que si con el ánimo de modificar una conducta socialmente indeseable - como la conducta violenta - esta se elimina de alguna manera, es necesario que el individuo aprenda una conducta alterna diferente, la cual pueda mostrar en sus relaciones con los demás. Esta sería una conducta opuesta a la violenta, es decir, la conducta pacífica. La conducta pacífica es diametralmente opuesta a la conducta violenta, y es socialmente aceptable, genera bienestar y convivencia pacífica en la comunidad. Por ejemplo, la violencia intrafamiliar, generalmente ejercida en el hogar por el adulto 
varón contra la mujer y/o los niños, se impone por la fuerza del victimario y la debilidad de las víctimas, pero el victimario tiende a repetir esa conducta violenta cada vez que quiere logar algo... iy lo logra! Ello hace que repita esa conducta cada vez con más frecuencia. Surge y se fortalece entonces un círculo vicioso de violencia: el victimario agrede y aprende a seguir agrediendo por las consecuencias satisfactorias de dominio para él. Por el contrario, las víctimas aprenden a someterse y soportar el castigo, pues al final logran que el victimario "proteja" a la familia. Así, la violencia se ejerce como mecanismo de resolución de conflictos en el hogar (Organización Panamericana de la Salud, 2012).

En tercer lugar, los observadores de la conducta violenta también aprenden a ser violentos. Bandura (1973) demostró que la conducta violenta se aprende por observación e imitación, y lo explica por la prevalencia de un tipo de reforzamiento social: el reforzamiento vicario o indirecto. No será necesario experimentar la violencia sino que basta observarla para aprender a ejecutarla, o padecerla. De ahí que hijos de padres violentos muy probablemente serán violentos en su propio hogar. Pero, igualmente, hijas de mujeres víctimas de la violencia intrafamiliar, muy probablemente también serán víctimas en su propio hogar.

Finalmente, cabe destacar también que la conducta violenta en la sociedad persiste debido a que las alternativas a ella han sido equívocas. A continuación se exponen algunas de ellas.

- La no-violencia. Doctrina y estrategia política inicialmente utilizada por Mahatma Gandhi a fin de doblegar a los británicos y solicitarles su salida de la India, y así sus ciudadanos pudieran escoger libremente sus autoridades y leyes de convivencia y desarrollo como país. Se destaca por su pasividad frente a la violencia del agresor. Como consecuencia, fueron masacrados con balas asesinas muchas personas, hombres, adultos mayores, mujeres y niños. El rechazo social y político de la masacre como consecuencia social inmediata de la sociedad hindú y de la comunidad internacional hizo que finalmente cesara la violencia, los británicos se retiraran y reconocieran la libertad y la dignidad de esta nación. Sin embargo, el precio fue demasiado alto en vidas humanas. Muchas otras personas han utilizado esta estrategia política de la no-violencia, resistiendo de manera estoica todo tipo de agresión y vejámenes a la dignidad humana.
Los afrodescendientes, con Martin Luther King (Estados Unidos) y Nelson Mandela (Sudáfrica), fueron los personajes más reconocidos. En Colombia, se recuerda no hace mucho tiempo (abril del 2002) este camino seguido por Guillermo Gaviria Correa, gobernador del departamento de Antioquia en ese entonces, quien con un grupo de sus dirigentes y líderes comunitarios marchó hacia las montañas con el fin de hablar con los guerrilleros y persuadirlos de su accionar. El resultado fue su retención y posterior asesinato. El movimiento se disolvió. De igual forma, muchos otros movimientos se han inspirado en esa estrategia frente al abuso y el poderío militar: recostarse en el piso, desnudarse, taparse la boca, entre otras manifestaciones, son las más comunes. El resultado: un comportamiento pasivo que no conduce sino a la imposición del violento y a la sumisión frente a la violencia. Cabe destacar nuevamente que este tipo de comportamiento social está imbuido de una mal entendida cultura occidental y cristiana vigente en nuestros países de "poner la otra mejilla", hasta que el otro se sacie. Lo importante en estos ejemplos es lo referente a la conducta social opuesta al comportamiento violento. De hecho, como se puede colegir, la conducta pasiva de la no-violencia no es la alternativa a la violencia, sino todo lo contrario, la fortalece; no es una conducta activa que construye o propone algo nuevo en la interacción humana. Por ello, tal vez, la sumisión de las víctimas en el hogar, en la escuela (bullying), y sus secuelas de depresión y finalmente suicidio.

- Aulas en Paz. Similar a otras alternativas como las de Comunidades de Paz, Aldeas en Paz, entre otras. Aulas en Paz es un proyecto que desarrolla la Universidad de los Andes en Colombia (Jimenez, Lleras y Nieto, 2010), como alternativa a la violencia escolar y bullying (violencia entre pares), en la que se muestran habilidades y comportamientos sociales diferentes a las violentas de un niño para que otros la observen y tengan probabilidades de repetirlas. Todo según los postulados de Bandura (1976).

Cabe señalar, en primer lugar, que hay un error de identificación del sujeto. No son las aulas las que necesitan estar en paz, son los niños los que necesitan aprender la conducta pacífica y así generar unas relaciones pacíficas en el aula de clases. El problema es, ¿cómo? El proyecto desarrolla habilidades sociales para la interacción entre los niños en el aula escolar, pero no conducta 
pacífica, pues al parecer es una categoría que no utilizan, ya que no la han definido ni caracterizado. El proyecto al parecer está mostrando buenos resultados, pero no se sabe a qué se debe: si al aprendizaje de una nueva conducta social pacífica moldeada en el ambiente escolar, o a un conjunto de habilidades para la interacción que no puede ser reproducida, aprendida ni enseñada socialmente con facilidad.

\section{La conducta violenta se aprende y es reforzada socialmente}

Una vez aprendida la conducta violenta, por reforzamiento operante o vicario, esta será reforzada socialmente y hará parte del repertorio de conducta específica del individuo, es decir, será una característica personal del mismo.

Siendo así, se puede considerar la conducta violenta como una conducta social aprendida socialmente, es decir, en interacción con otros seres humanos, en una comunidad humana. De acuerdo con Bandura, el comportamiento agresivo (o violento, mejor), se aprende socialmente mediante la observación y la imitación, debido a que es reforzado de manera vicaria.

En primer lugar, la conducta violenta se refuerza debido a sus consecuencias inmediatas: el agresor logra lo que se propone y eso lo satisface, emocional, laboral o socialmente. Ello hará que el agresor ejerza violencia cada vez que lo necesite en sus interacciones con las demás personas. Esto, muy a pesar de que las consecuencias mediatas de sus actos lo puedan conducir a consecuencias negativas para él (encarcelamiento, restricción de sus derechos sociales, abandono familiar, expulsión de las aulas, entre otros). Tal como sucede con el hábito de fumar, el fumador consume diariamente a sabiendas de los efectos nocivos comprobados para la salud del fumador, entre ellos, el cáncer pulmonar. Pero su consumo se explica por el placer inmediato del fumar: reducción de la ansiedad, evitación del sobrepeso, manipulación social, entre otros. Dicho hábito hace parte de su repertorio conductual que lo lleva a consumir cada vez que se presenten situaciones sociales similares al consumo. Una vez adquiera dependencia física a sus componentes, habrá caído irremediablemente en este hábito, del cual será muy difícil salir.

En segundo lugar, la conducta violenta es de alguna manera aprobada socialmente en la cultura occidental, caracterizada por ser machista, patriarcal y heterosexual (Aller, 1996). En esta se acepta el predominio no solo del hombre sobre la mujer y los niños, sino de todo tipo de conducta machista, ya sea en el hombre, la mujer o los niños, representada por la fuerza, la violencia, la imposición sobre el otro y el control emocional en situaciones difíciles. Por ello, a pesar de todo, la familia suele vivir en medio de la violencia intrafamiliar, principalmente ejercida por el padre, a quien se le otorga socialmente el derecho de reprimir a su prole y a su mujer. Igualmente, la población suele aprobar socialmente el uso de la fuerza del Estado por medio de más "pie de fuerza militar", a quien se le ha dado el derecho de ejercer violencia legal contra quienes de diversas maneras se oponen o amenazan el statu quo. De esta manera, se construyen muchas más cárceles y cementerios que escuelas, viviendas y hospitales.

En estas condiciones individuales y sociales, es muy difícil revertir la conducta violenta en la sociedad ejerciendo una mayor violencia. "Ojo por ojo...y la humanidad quedará ciega”, sentenció Gandhi (1972). Por ello, las políticas de Estado con relación a la convivencia social, deben modificarse radicalmente con el fin de promover la conducta pacífica y no la violenta, tanto en la sociedad como en sus instituciones, principalmente en la escuela y la familia.

\section{La conducta pacífica como alternativa también se aprende y se mantiene por reforzamiento social: ¿cómo se explica? ¿cómo enseñarla?}

Algo que se descubrió, pero que no se explicó suficientemente en el experimento de Bandura (1973), fue precisamente la conducta pacífica. Bandura descubrió cómo los niños que observaron a otros golpear un muñeco, también lo golpean cuando tienen una oportunidad similar a la observada, sin importar si son niños o niñas. Sin embargo, también halló que los niños que observan a otro niño (modelo) acariciar un objeto, también lo acarician y no lo golpean, es más, lo defienden de aquel niño que busca golpearlo. Los niños hablaban entre sí, se miraban, sonreían, acariciaban el objeto sin arrebatárselo entre sí, y se ubicaban en sitios cómodos del ambiente, sin atropellarse unos a otros. Bandura le llamó a esta conducta alterna "conducta pacífica". Aunque este autor alcanzó a manifestar que la conducta pacífica es una conducta social, la cual al igual que la conducta violenta se puede aprender socialmente por reforzamiento vicario de modelos significativos para 
los niños, y sostenerse por reforzamiento social solo si la comunidad a la que pertenece el niño la valora socialmente. Sin embargo, dados los objetivos de su proyecto, Bandura solo desarrolló la explicación de la conducta violenta como un comportamiento socialmente aprendido y reforzado de manera vicaria.

\section{Conducta pacífica, alternativa de la psicología positiva para la salud, educación y felicidad}

De acuerdo con Bandura (1973), la conducta pacífica se aprende de la misma manera que la conducta violenta, pues es una conducta social como aquella.

Desde la perspectiva de Seligman y Csikszentmihalyi (2000), la psicología como ciencia se ha centrado demasiado y por mucho tiempo en lo patológico, en los problemas del comportamiento humano. Desde la Segunda Guerra Mundial, en la que los psicólogos se dedicaron a seleccionar reclutas para la guerra enfatizando en sus debilidades más que en sus fortalezas, la psicología en general y la psicología clínica en particular, se han dedicado a estudiar los problemas y las anormalidades del ser humano. Seligman llama la atención de este hecho y desde 1990 planteó que la psicología debe volver a sus raíces iniciales. Debe, por tanto, estudiar el comportamiento de las personas a fin de promover su salud y bienestar, indicando que esa parte positiva de las personas es lo más estable de su conducta, y remarcando que la alteración o anormalidad es situacional. De acuerdo con ello, Seligman es reconocido como el fundador y representante de la moderna psicología positiva, la cual postula precisamente que la psicología debe estudiar los aspectos positivos más estables que presentan las personas, tales como optimismo, satisfacción, resiliencia, bienestar psicológico, engagement o vinculación psicológica al trabajo, así como felicidad.

Desde esta perspectiva, el estudio de la conducta pacífica entra en los límites de la psicología positiva, al definirse como una conducta deseable, saludable, humana y humanizante, que necesariamente lleva a la salud y la felicidad, puesto que una convivencia pacífica genera altas satisfacciones individuales y sociales.

Si se practica y valora socialmente la conducta pacífica, entonces tendrá grandes probabilidades de fortalecerse, generalizarse y expandirse ilimitadamente, favoreciendo el desarrollo humano en sociedad, pues, como se ha afirmado, según Skinner
(1981) toda conducta reforzada de manera contingente tendrá grandes probabilidades de repetirse e incrementarse.

\section{El entrenamiento de la conducta pacífica}

Sin embargo, ¿qué es la conducta pacífica? ¿Cómo se define? ¿Se puede enseñar? ¿Se puede aprender?

La conducta pacífica es una conducta opuesta a la conducta violenta. Y, dado que la conducta violenta no es socialmente deseable ni positiva, la conducta pacífica, por el contrario, es socialmente deseable, positiva y generadora de sana convivencia en sociedad. Por tanto, apetecida como generadora de una sana convivencia social. Al señalar que la conducta pacífica puede ser socialmente aprendida como la conducta violenta, Bandura (1973) destaca la importancia del reforzamiento social de esta conducta, tanto en el aula, como en la familia y la sociedad.

Cuando un padre de familia, profesor o dirigente social llama la atención por su comportamiento violento a un niño, joven o adulto, suele exigirle el cese de su conducta: "¡No seas violento! No golpees! No grites! No insultes! No amenaces! No... no... no... no".

Si se detalla esta situación, en el caso que el individuo hace caso a la orden verbal, ¿qué hace el individuo finalmente? Cesa su actividad, queda pasivo, no hace nada, como si ya no tuviera conducta que mostrar. ¿Es esa la conducta "pacífica" de la que se habla? Definitivamente no.

Al realizar una precisión de categoria, la conducta deseable en el ser humano no es la conducta pasiva (no es deseable ni real), ni la conducta no-violenta (por ser indefinida y difícil de enseñar).

En primer lugar cabe precisar lo que no es la conducta pacífica: no es pasiva, sometida, dependiente, sumisa (afecta las relaciones interpersonales deseables del niño y del futuro adulto). En cambio, es activa, autónoma, dinámica, verbal, efectiva y humana.

- Es activa porque es una forma de actuar, es una decisión individual, es trasformadora de las relaciones sociales (prosocial); exige cambios en el otro (persona o institución), transforma al otro y al entorno social humano.

- Es autónoma y dinámica porque es una decisión del sí-mismo, el locus de control se encuentra en la persona. Es dinámica porque es de uso constante sujeta a las interacciones sociales, lo cual implica cambios que respondan al entorno social 
de la persona, así como promueve y refuerza su uso como opuesta a la conducta violenta y exige el cambio del otro, persona o institución en su uso constante.

- Es verbal, efectiva y humana porque es dialogante, recíproca, asertiva, propositiva, aspectos propios del diálogo humano; es efectiva porque promueve la concertación dialogante en los acuerdos y desacuerdos en la interacción con otros; y porque logra objetivos sociales de comprensión, aceptación y decisión de común acuerdo. Es humana porque se basa en el uso del lenguaje humano y promueve su humanización (Skinner, 1981; Hayes, Barnes-Holmes y Roche, 2001).

\section{Algunos principios del aprendizaje involucrados en la nueva conducta pacífica}

El aprendizaje, la enseñanza y la generalización social de la conducta pacífica es posible por la vigencia de los siguientes principios del aprendizaje:

- Principio del reforzamiento (Skinner, 1994; Bandura, 1976): toda conducta que es reforzada de manera contingente tiende a repetirse e incrementarse.

- Principio de discriminación y generalización: toda conducta aprendida suele presentarse en situaciones específicas y tiende a generalizarse en dichas situaciones diferenciándose de otro tipo de conductas.

- Principio de extinción: una conducta no reforzada de manera contingente tiende a debilitarse y extinguirse.

- Principio de castigo: una conducta que es seguida de un castigo intenso de manera contingente tiende a eliminarse.

La cuestión central es que al eliminar una conducta, como la conducta violenta en los niños y jóvenes, debe instaurarse otra opuesta mediante el aprendizaje por reforzamiento: la conducta pacífica.

\section{Desarrollo de la conducta pacífica para promover un estilo de vida saludable, el bienestar psicológico $y$ un estado de felicidad}

La conducta pacífica, al igual que la violenta, es aprendida y puede ser enseñable y promovida socialmente.
Los niños que la aprenden pueden extender su conducta pacífica aprendida a los años posteriores, es decir, a la adolescencia y a la vejez. Es más, los niños que aprenden la conducta pacífica, al igual que la conducta violenta, constituirán una sociedad pacífica y una cultura de paz, la cual garantizaría la sana convivencia, el bienestar y la felicidad humana, objetos de estudio y finalidad de la psicología positiva.

De acuerdo con la Organización Mundial de la Salud (1986), la felicidad no es una meta a alcanzar al final de la vida, sino más bien, al igual que la salud, es un recurso para la vida, es propio de la vida humana, es su sentido y orientación vital. No se puede concebir el desarrollo integral del ser humano al margen de la felicidad y el bienestar en cada una de sus acciones. La prevalencia de la conducta violenta no es sino una manifestación de su degradación, pues de acuerdo con Savater (1997) "el hombre se va humanizando, a través de la educación y la cultura”, lo cual no puede ser otra cosa que la prevalencia de la conducta pacífica y una verdadera cultura de paz que la promueva y fortalezca.

Esto requiere una reformulación de las políticas de salud y educación del Estado, a fin de garantizar el pleno ejercicio de sus derechos humanos, entre ellos el más fundamental: el derecho a la vida digna, a la vida humana.

\section{Conclusión}

La sociedad occidental en particular, dentro de la cual se inscriben las sociedades latinoamericanas, y gran parte de la humanidad en general, ha equivocado el camino al tratar de construir estas sociedades priorizando el control, el poder y el capital. De seguir así, la conducta violenta, social e individual, será una epidemia incontrolable que llevará a la humanidad a su propia destrucción. En el camino, tal vez media humanidad armada tendrá que controlar a otra media humanidad desarmada sufriente.

Desde la ciencia psicológica, a partir de este escrito es posible señalar la importancia de promover el aprendizaje de la conducta pacífica como única forma válida para lograr la sana convivencia social, con bienestar y felicidad. El Estado debe reformular o incluir dentro de sus políticas públicas - en especial de educación y salud-, la enseñanza y el aprendizaje de la conducta pacífica que permita gestar una verdadera cultura de paz, que la promueva y refuerce socialmente. 
La vigencia de la conducta pacífica en las interacciones humanas implica respeto, responsabilidad, genera altruismo y solidaridad, valores necesarios para la convivencia y el desarrollo social humano. Ello implica al mismo tiempo un cambio en los adultos (padres de familia, profesores, autoridades, instituciones y Estado), hacia la humanización de la sociedad, hacia unas ciudades e instituciones saludables.

Los aportes de la psicología cognitivo-conductual de Bandura (1976), del condicionamiento operante de Skinner (1981); y los aportes recientes sobre el comportamiento verbal de Hayes et al. (2001); así como de la psicología positiva de Seligman, M. (2002), pueden servir de soporte científico, con fundamento experimental, a fin de propiciar este urgente cambio de la conducta humana. Desde los hogares, pasando por la escuela y otras instituciones educativas, hasta las empresas y las organizaciones sociales, deben comprometerse a ello.

No hay que olvidar como, en concordancia con las ideas de Jean-Jacques Rousseau (1866), quien sostenía la teoría según la cual el hombre era naturalmente bueno, la sociedad corrompía esta bondad y, por lo tanto, la persona no nacía perversa sino que se hacía perversa, de manera que era necesario volver a la virtud primitiva (es decir, la actitud de bondad o de maldad es fruto del medio social en el cual se desarrolla el individuo), Albert Bandura consideró que el comportamiento humano violento, más que ser genético o hereditario, es un fenómeno adquirido por medio de la observación e imitación. Puesto que la conducta violenta de los hombres no es una condición humana sino una respuesta, el hombre no nace con un carácter violento, sino con un sistema muy organizado de tendencias hacia el crecimiento y el desarrollo de su ambiente, de comprensión y cooperación (Bandura, 1976).

Finalmente, cabe señalar la importancia y necesidad de impulsar el desarrollo de proyectos de investigación científica sobre este tema, a fin de reorientar la formación integral de nuestros niños y adolescentes principalmente, y a su vez generar nuevos conocimientos para la comprensión de la conducta pacífica/violenta, desde y para la ciencia psicológi$\mathrm{ca}$, con el concurso de otras ciencias afines como la educación y la salud. Queda, pues, un nuevo camino por recorrer.

\section{Referencias}

Aller, L. M. (1996). Pedagogía de la sexualidad humana. En L. García de Maya y I. P. Sánchez (Comps.), Sexualidad y Educación (pp. 26-45). Bogotá: Ed. Magisterio.

Bandura, A. (1973). Aggression. A social learning analysis. New Jersey: Prentice Hall.

Bandura, A. (1976). Teoría del aprendizaje social. Madrid: Espasa Calpe.

Gandhi, M. (1972). Historia de mis experiencias con la Verdad (Autobiografía).Madrid: Gaia.

Hayes, S. C., Barnes-Holmes, D. y Roche, B. (Eds.). (2001). Relational Frame Theory: A Post-Skinnerian account of human language and cognition. New York: Plenum Press.

Jiménez, M., Lleras, J. y Nieto, A. (2010). La paz nace en las aulas: evaluación del programa de reducción de la violencia en Colombia. Educ., 13(3), 347-359. Recuperado de: http://www.scielo.org.co/pdf/eded/v13n3/ v13n3a03

Organización Mundial de la Salud. [oms]. (1986). Otawa Charter for Health Promotion. Carta de Otawa para la Promoción de la salud. Otawa. Canadá. Recuperado de: http://www.fmed.uba.ar/depto/toxicol/carta.pdf

Organización Panamericana de la Salud. (2012). La violencia intrafamiliar y el maltrato a la infancia en Colombia. Recuperado de: http://www.paho.org/chi/index. php?option $=$ com_docman \&task=doc_view\&gid $=123 \&$ Itemid

Rousseau, J. J. (1866). Emilio o de la Educación. Oxford: Universidad de Oxford.

Savater, F. (1997). El valor de educar. Barcelona: Ed. Ariel.

Seligman, M. (2002). Authentic Happiness: Using the New Positive Psychology to Realize Your Potential for Lasting Fulfillment. New York: Free Press.

Seligman, M.yCsikszentmihalyi, M. (2000). PositivePsychology: An Introduction. American Psychologist, 55(1), 5-14. doi: http://dx.doi.org/10.1037/0003-066X.55.1.5

Skinner, B. F. (1953). Science and Human Behavior. New York: Free Press.

Skinner, B. F. (1981). Conducta verbal. México: Trillas.

Skinner, B. F. (1994). Sobre el conductismo. Buenos Aires: Planeta. Recuperado de: http://www.conductitlan.net/ b_f_skinner/b_f_skinner_sobre_el_conductismo.pdf 